\title{
A Balloon Lens: \\ Acoustic Scattering from a Penetrable Sphere
}

Derek Thomas

Capstone Project Physics 492R

Advisor: Kent L. Gee

August 13, 2007 


\begin{abstract}
A balloon filled with a gas that has a different sound speed than that of air has been used by science teachers in the past as an "acoustic lens." One purpose of the lens is to show refraction of sound waves as an analogy to geometric optics [H. Kruglak and C. C. Kruse, Am. J. Phys. 8, 260-261 (1940)]. This paper discusses the physics involved with the balloon lens demonstration. In order to determine the validity of a gas-filled balloon as a classroom demonstration of an acoustic lens and to understand the corresponding phenomena, the problem is considered analytically, numerically, and experimentally. The results show that although a geometric analogy is a valid first order approximation, scattering theory is often required to fully understand the observed phenomena. Thus, this demonstration can be adapted to a wide range of students, from those learning basic principles of refraction to mathematically advanced students studying scattering.
\end{abstract}




\section{Contents}

1 Introduction $\quad 1$

2 Experimental Analysis and Results 2

3 Numerical Methods and Results 2

4 Analytical Methods and Results 4

4.1 Boundary Conditions . . . . . . . . . . . . . . . . . . 7

4.2 Green's Function . . . . . . . . . . . . . . . . . . . . . . . . . . . . . . . . . .

4.3 Scattering Theory . . . . . . . . . . . . . . . . 8

5 Discussion and Conclusion $\quad 10$

\section{List of Figures}

1 Region $0.22 \mathrm{~m}$ by $0.3 \mathrm{~m}$ in front of balloon at approximately $3000 \mathrm{~Hz}$ as observed experimentally. . . . . . . . . . . . . . . . . . . 12

2 Region $0.22 \mathrm{~m}$ by $0.3 \mathrm{~m}$ in front of balloon at approximately $5000 \mathrm{~Hz}$ as observed experimentally. . . . . . . . . . . . . . . . 13

3 Region $0.22 \mathrm{~m}$ by $0.3 \mathrm{~m}$ in front of balloon at approximately $3000 \mathrm{~Hz}$ as predicted by a staggered leapfrog method. . . . . . . . . . . . . . . . 14

4 Region $0.22 \mathrm{~m}$ by $0.3 \mathrm{~m}$ in front of balloon at approximately $5000 \mathrm{~Hz}$ as predicted by a staggered leapfrog method. . . . . . . . . . . . . . 15

5 Region $0.22 \mathrm{~m}$ by $0.3 \mathrm{~m}$ in front of balloon at $3000 \mathrm{~Hz}$ as predicted using an analytical series solution. . . . . . . . . . . . . . . . . 16

6 Region $0.22 \mathrm{~m}$ by $0.3 \mathrm{~m}$ in front of balloon at $5000 \mathrm{~Hz}$ as predicted using an analytical series solution. . . . . . . . . . . . . . . . 17

$7 \quad$ Spherical Coordinate System . . . . . . . . . . . . . . . . . . . 18 


\section{Introduction}

The balloon lens is suggested as a demonstration on many websites and used in various museums. Unfortunately, many of the explanations available to teachers are either overly simplistic, confusing or wrong. The purpose of this paper is to present a correct explanation of the physics of a balloon lens along with experimental, numerical, and analytical results in order to provide teachers with an adequate resource to use this demonstration in the classroom.

The speed of sound $c$ in a gas may be written as

$$
c=\sqrt{\frac{\gamma P_{0}}{\rho_{0}}}
$$

where $\gamma$ is the ratio of specific heats, $P_{0}$ is the equilibrium pressure (atmospheric), and $\rho_{0}$ is the equilibrium density. Clearly, as the density increases, the speed of sound decreases. Fermat's principle states that a wave will travel the shortest possible time-path. This gives rise to Snell's law, which states that the ray parallel to the direction of wave propagation will bend at an interface between two media with different sound speeds. The angle the ray will bend away from the normal is given by

$$
\frac{1}{c_{1}} \sin \theta_{1}=\frac{1}{c_{2}} \sin \theta_{2}
$$

where $\theta_{1}$ is the angle from the surface normal of the incident wave and $c_{1}$ is the wave speed on the incident side of the interface; $\theta_{2}$ is the angle from the surface normal of the transmitted wave and $c_{2}$ is the wave speed on the transmitted side [1, p.267]. By considering the path traveled by multiple rays due to a source or incident plane wave, it is possible to determine the focal point of a lens. This is the simplest explanation and the one most suited for discussion in an introductory setting. However, the ray tracing method is inaccurate for audible sound because the derivation of Snell's law assumes that the wavelength is much smaller than the dimensions of the refracting object. This is a valid assumption for an optical lens, however, to achieve a similar ratio of lens dimension to wavelength with audible sound having a frequency of $1000 \mathrm{~Hz}$ would require a lens 10-20 km (6.2-12.4 miles) in diameter. The prediction found using ray tracing is better if ultrasound is used, however, many teaching resources do not consider this. Also, using ultrasound eliminates the hands-on nature of the demonstration. The analogy to an optical lens and the ray tracing approach provides a firstorder approximation, but to accurately predict the behavior of an acoustic lens requires more advanced methods. This paper is meant to fill an apparent void for physics teachers and students. Experimental results that illustrate the characteristics of the scattered field are presented. Numerical methods appropriate for a junior-senior level course in computational physics are presented here along with analytical methods that are typically introduced at 
a graduate level. The balloon is often used as a lens between two people, one speaker and one receiver. This configuration could be considered using a point source at an arbitrary location, however, because the analytical calculations are simpler for an incident plane wave, only the plane wave case is considered here. It is reasonable to assume that the point source would produce similar results.

\section{Experimental Analysis and Results}

The behavior of the balloon as a lens was analyzed in an anechoic chamber to reduce interference due to reflection. In order to approximate a plane wave, the source was placed as far from the balloon as possible.

To resolve the details of the "focal" region of the balloon lens, a linear array of 23 GRAS $1 / 4$ inch microphones spaced at $1 / 2$ inch intervals was used to scan from approximately 5 centimeters out to 48 centimeters from the balloon. The balloon was excited by a white noise signal from a Mackie HR824 studio monitor. The balloon was approximately spherical with a radius of 0.3 meters and was placed approximately 5 meters from the source in an anechoic chamber. The resulting data was filtered and post-processed using LabView and Matlab. The resulting arrays of sound pressure level values (dB ref $20 \mu \mathrm{Pa}$ ) were plotted using Matlab.

As can be seen in Figure 1, at $3000 \mathrm{~Hz}$ there is a peak in the sound pressure level (SPL) that seems to correspond to a focal point. However, there are two small peaks on the edges of the scanning region that are not predicted by treating the balloon as a simple acoustic lens. These lobes become more pronounced and more numerous as the frequency increases, as is apparent in Figure 2 which is a plot of the same region at $5000 \mathrm{~Hz}$.

\section{$3 \quad$ Numerical Methods and Results}

A simple numerical model may be constructed using basic numerical techniques for solving partial differential equations. A staggered leapfrog method uses a second order approximation of the operator

$$
\frac{\partial^{2}}{\partial x^{2}}
$$

in space and time. Using this approximation it is possible to solve for the future values of the field in terms of the previous values. This method also has the advantage of producing an animation as it computes, thus allowing the student to observe the field as it evolves.

One assumption simplifies the numerical computation significantly. By assuming that the problem is axially symmetric, the problem may be reduced from three dimensions to two. Although this assumption actually reduces the problem to the case of an infinite, penetrable cylinder instead of a penetrable sphere, and thus prevents quantitative comparison between analytical, experimental and numerical data, it reduces computational time drasti- 
cally and permits qualitative comparisons of features such as the number of lobes and basic characteristics of the field.

Solving this problem analytically requires the solution of the scalar wave equation,

$$
\nabla^{2} p=\frac{1}{c^{2}} \frac{\partial^{2} p}{\partial t^{2}}
$$

where $c$ is the speed of sound and $p$ is the pressure In two-dimensional cartesian coordinates, this equation becomes

$$
\frac{1}{c^{2}} \frac{\partial^{2} p}{\partial t^{2}}=\frac{\partial^{2} p}{\partial x^{2}}+\frac{\partial^{2} p}{\partial y^{2}}
$$

which can be solved in a straight forward manner using a staggered leapfrog method.

\section{Staggered Leapfrog Method}

In the staggered leapfrog approach a finite difference is used in both time and space. The first approximations to the second derivatives in each dimension are

$$
\begin{aligned}
\frac{\partial^{2} p}{\partial t^{2}} & \approx \frac{p_{i, j}^{n+1}-2 p_{i, j}^{n}+p_{i, j}^{n-1}}{\tau^{2}} \\
\frac{\partial^{2} p}{\partial x^{2}} & \approx \frac{p_{i+1, j}^{n}-2 p_{i, j}^{n}+p_{i-1, j}^{n}}{h_{x}^{2}} \\
\frac{\partial^{2} p}{\partial y^{2}} & \approx \frac{p_{i, j+1}^{n}-2 p_{i, j}^{n}+p_{i, j-1}^{n}}{h_{y}^{2}}
\end{aligned}
$$

where $\tau$ is the time step and $h_{x}$ and $h_{y}$ are the spatial steps in the $x$ and $y$ dimensions. The indices $i$ and $j$ refer to the row and column of the two-dimensional spatial array describing pressure and $n$ is the time index. These approximations are then used in the wave equation

$$
\frac{1}{c^{2}} \frac{p_{i, j}^{n+1}-2 p_{i, j}^{n}+p_{i, j}^{n-1}}{\tau^{2}}=\frac{p_{i+1, j}^{n}-2 p_{i, j}^{n}+p_{i-1, j}^{n}}{h_{x}^{2}}+\frac{p_{i, j+1}^{n}-2 p_{i, j}^{n}+p_{i, j-1}^{n}}{h_{y}^{2}} .
$$

The staggered leapfrog algorithm uses the previous values to determine the next value. Solving Eq.(4) for the future value $p_{i, j}^{n+1}$ yields

$$
p_{i, j}^{n+1}=2 p_{i, j}^{n}-p_{i, j}^{n-1}+\frac{c^{2} \tau^{2}}{h_{x}^{2}}\left(p_{i+1, j}^{n}-2 p_{i, j}^{n}+p_{i-1, j}^{n}\right)+\frac{c^{2} \tau^{2}}{h_{y}^{2}}\left(p_{i, j+1}^{n}-2 p_{i, j}^{n}+p_{i, j-1}^{n}\right) .
$$

The presence of the balloon can be modeled by including a circular region with a different sound speed in the center of the computational domain.

It is important to note the limitations of this method. First, the time step size $\tau$ and the spatial step sizes $h_{x}$ and $h_{y}$ must be chosen so that

$$
\tau<\frac{h_{x}}{c}
$$


and

$$
\tau<\frac{h_{y}}{c} .
$$

This is known as the Courant-Fridrichs-Levy condition or CFL condition, and if it is not satisfied, the algorithm becomes unstable. Also, because it is difficult to implement a radiation condition at the boundary of the grid, any wave incident on the boundary will be reflected, which can cause erroneous results. In order to avoid this problem, it is necessary to generate a grid large enough to allow the simulation to proceed without error until the desired results are obtained. The simulation can then be terminated. Another limitation that must be considered is due to the reduction from three dimensions to two. This means that the results should be spatially accurate, but the amplitudes may not correlate with the three-dimensional case. This limits analysis to a qualitative comparison.

In order to compare the numerical results to the experiment, the numerical grid was sampled at points corresponding to "microphones" and these results were time averaged to produce the plots in Figures 3 and 4 . When Figures 3 and 4 are compared with Figures 1 and 2 , it is apparent that the results are very similar, and that the numerical model predicts the lobing behavior observed in the experimental results.

\section{Analytical Methods and Results}

Although the balloon lens demonstration has been used primarily in introductory physics courses, Mie scattering theory is necessary in order to completely describe the phenomenon analytically. Typically, this is a topic of graduate courses in mathematical methods in physics or electrics and magnetism. A brief discussion of the necessary methods is included. For this problem, a series approach is adequate, however, for a more general approach using the Helmholtz integral, see Kress [2].

Similar to the previous sections, only the case of a plane wave incident on a spherical balloon is considered. The pressure field due to small amplitude excitations of the medium is found by solving the acoustic wave equation

$$
\nabla^{2} p=\frac{1}{c^{2}} \frac{\partial^{2} p}{\partial t^{2}}
$$

This equation can be derived from basic properties of the medium ??, however, the derivation is omitted at this time. If time-harmonic excitation is presumed, then it is reasonable to assume that the pressure is of the form $p(\mathbf{x}, t)=\Re\left\{p(\mathbf{x}) e^{-i \omega t}\right\}$ where $i$ is the imaginary number $i=\sqrt{-1}$. Following this assumption the time-dependent wave equation is reduced to the time-independent Helmholtz equation

$$
\left(\nabla^{2}+k^{2}\right) p(\mathbf{x})=0
$$

where $k$ follows the relation

$$
k^{2}=\left(\frac{2 \pi}{\lambda}\right)^{2}=\frac{\omega^{2}}{c^{2}}
$$


where $c$ is the thermodynamic speed of sound, $\lambda$ is the wavelength and $\omega$ is the angular frequency.

\section{Solutions in Spherical Coordinates}

In this paper $r$ will represent the radial distance from the origin, $\phi$ is the azimuthal angle from the $x$-axis in a right handed coordinate system, and $\theta$ is the polar angle down from the $z$-axis. This is known as a spherical coordinate system. In spherical coordinates, the Laplacian operator $\nabla^{2}$ takes the form

$$
\nabla^{2}=\frac{1}{r^{2}} \frac{\partial}{\partial r}\left(r^{2} \frac{\partial}{\partial r}\right)+\frac{1}{r^{2} \sin \theta} \frac{\partial^{2}}{\partial \phi^{2}}+\frac{1}{r^{2} \sin \theta} \frac{\partial}{\partial \theta}\left(\sin \theta \frac{\partial}{\partial \theta}\right)
$$

The Helmholtz equation in spherical coordinates is then

$$
\left(\frac{1}{r^{2}} \frac{\partial}{\partial r}\left(r^{2} \frac{\partial}{\partial r}\right)+\frac{1}{r^{2} \sin \theta} \frac{\partial^{2}}{\partial \phi^{2}}+\frac{1}{r^{2} \sin \theta} \frac{\partial}{\partial \theta}\left(\sin \theta \frac{\partial}{\partial \theta}\right)+k^{2}\right) p(r, \phi, \theta)=0
$$

This equation can be solved by the separation of variables, letting $p(r, \phi, \theta)=R(r) \Phi(\phi) \Theta(\theta)$.

\section{Spherical Harmonics}

The dependence on the azimuthal angle $\phi$ is found by solving the $\phi$-dependent portion of the Helmholtz equation,

$$
\frac{1}{\Phi(\phi)} \frac{d^{2}}{d \phi^{2}} \Phi(\phi)=-m^{2}
$$

where $m$ is an integer, a restriction on $m$ made with some foresight. It is simple to show that the general solution to this equation is composed of $\sin (m \phi)$ and $\cos (m \phi)$ or alternately of the complex exponentials $e^{i m \phi}$ and $e^{-i m \phi}$.

Similarly, the dependence on the polar angle $\theta$ is found by solving

$$
\frac{1}{\sin \theta} \frac{d}{d \theta}\left(\sin \theta \frac{d}{d \theta} \Theta(\theta)\right)+\left(n(n+1)-\frac{m^{2}}{\sin ^{2} \theta}\right) \Theta(\theta)=0 .
$$

Where $m$ and $n$ are integers and it is required that $|m| \leq n$. This is the associated Legendre equation which has solutions $P_{n}^{m}(\cos \theta)$ and $Q_{n}^{m}(\cos \theta)$ that are known as the associated Legendre polynomials. Since $Q_{m}^{n}$ diverges for arguments equal to \pm 1 it will not be included in the solution. 
Since separation of variables was used to solve the Helmholtz equation, the total angular dependence of the pressure field is given by

$$
\Phi(\phi) \Theta(\theta)=A_{m, n} P_{n}^{m}(\cos \theta) e^{i m \phi}
$$

where $A_{m, n}$ is a constant, $n$ is an integer, and $m$ ranges from $-n$ to $n$. Traditionally the angular parts of the solutions to the Helmholtz equation are defined as the spherical harmonics

$$
Y_{n}^{m}(\phi, \theta)=\sqrt{\frac{2 n+1}{4 \pi} \frac{(n-m) !}{(n+m) !}} P_{n}^{m}(\cos \theta) e^{i m \phi} .
$$

Thus, equation 12 becomes

$$
\Phi(\phi) \Theta(\theta)=B_{m, n} Y_{n}^{m}(\theta, \phi)
$$

where

$$
B_{m, n}=A_{m, n}\left(\frac{2 n+1}{4 \pi} \frac{(n-m) !}{(n+m) !}\right)^{-1 / 2}
$$

\section{Spherical Bessel Functions}

It is possible to show that the radial portion of the Helmholtz equation in spherical coordinates is

$$
r^{2} \frac{d^{2}}{d r^{2}} R(r)+2 r \frac{d}{d r} R(r)+\left[k^{2} r^{2}-n(n+1)\right] R(r)=0 .
$$

The solutions to this equation are known as spherical Bessel functions of the first and second kind $j_{n}(k r)$ and $y_{n}(k r)[3$, p. 596]. These are related to the Bessel functions of the first and second kind by

$$
j_{m}(k r)=\sqrt{\frac{\pi}{2 k r}} J_{n+\frac{1}{2}}(k r)
$$

and

$$
y_{m}(k r)=\sqrt{\frac{\pi}{2 k r}} Y_{n+\frac{1}{2}}(k r) .
$$

Another formulation of the radial solution uses the spherical Hankel functions of the first and second kind which are related to the spherical Bessel functions as follows,

$$
h_{n}^{(1)}(k r)=j_{n}(k r)+i y_{n}(k r)
$$

and

$$
h_{n}^{(2)}(k r)=j_{n}(k r)-i y_{n}(k r) .
$$

The Hankel function of the first kind corresponds to spherical waves propagating outward from the origin, whereas the Hankel function of the second kind corresponds to propagating waves traveling inward, toward the origin. 
Combining the angular and radial portions of the solution results in the general solution,

$$
p(r, \phi, \theta)=\tilde{A}_{m, n} h_{n}^{(1)}(k r) Y_{m}^{n}(\phi, \theta)
$$

where $\tilde{A}_{m, n}$ is a constant determined by the boundary conditions. Note that equation 20 is still a solution to the differential equation independent of the kind of spherical Bessel or Hankel function used. The Hankel function and the Bessel function of the second kind both diverge at the origin, as opposed to the Bessel function of the first kind which is bounded over $\mathbb{R}^{3}$. The boundary conditions determine which type of equation is appropriate. It is also important to note that the solutions to the homogeneous Helmholtz equation form an orthogonal basis set of eigenfunctions for a spherical coordinate system.

\subsection{Boundary Conditions}

After a general solution has been found, boundary conditions are necessary to produce a solution unique to the problem at hand. For the case of an acoustic scatterer in free space, the boundaries are at the surface of the sphere, at the origin, and the limit as $r$ goes to infinity.

\section{Sommerfeld Radiation Condition and Boundedness Conditions}

It is intuitive to assume that the field radiating from a finite source, or alternately, the field scattered by a finite body goes to zero as the distance from the source approaches infinity. Mathematically, this is described by the Sommerfeld radiation condition,

$$
\lim _{r \rightarrow \infty}\left[r\left(\frac{\partial p}{\partial r}+\frac{1}{c} \frac{\partial p}{\partial t}\right)\right]=0 .
$$

Under the assumption of constant frequency time-harmonic excitation $\left(p(\mathbf{r}, t)=\Re\left\{p(\mathbf{r}) e^{-i \omega t}\right\}\right)$, equation 21 becomes

$$
\lim _{r \rightarrow \infty}\left[r\left(\frac{\partial p}{\partial r}-i k p\right)\right]=0
$$

see $[4$, p. 178].

Another condition on the pressure field is known as the boundedness condition. This condition requires that the pressure field remain bounded or finite at the origin. When applied, the Sommerfeld radiation condition and the boundedness condition determine what type of function is appropriate to describe the radial dependence of the pressure field $p$. The spherical Hankel functions satisfy the Sommerfeld radiation condition but fail to satisfy the boundedness condition. Thus, they are valid for all space exterior to an arbitrary sphere at the origin, making them useful for describing a scattered field. On the other hand, it can be shown that the spherical Bessel function of the first kind, $j_{m}$, fails to satisfy the Sommerfeld condition but does fulfill the boundedness condition. Thus, it is valid for the interior of an arbitrary sphere located at the origin. 


\section{Surface Conditions}

The remaining boundary is the surface of the scattering object. This boundary condition relates the incident wave to the scattered field and is determined by the properties of the scatterer. For a rigid boundary, the normal particle velocity is goes to zero. For a "soft" scatterer, i.e. a pressure release boundary, the pressure goes to zero. For the case of a penetrable scatterer, a few assumptions must be made before boundary conditions may be determined. The first assumption is that the layer separating the interior medium from the exterior medium is sufficiently thin that its presence may be ignored. Also, it is assumed that both media are continuous and that neither medium can exert transverse forces. Following these assumptions, the pressure and the normal velocity must be continuous across the boundary. This means that

$$
\begin{gathered}
p_{i}+p_{s}=p_{d} \\
\frac{1}{\rho} \frac{\partial}{\partial \hat{n}}\left(p_{i}+p_{s}\right)=\frac{1}{\rho_{d}} \frac{\partial p_{d}}{\partial \hat{n}}
\end{gathered}
$$

where use has been made of Euler's equation

$$
\nabla p=-\rho \frac{\partial \mathbf{u}}{\partial t}
$$

where $\mathbf{u}$ represents the particle velocity. Following the assumption of time-harmonic excitation and considering only normal velocity, equation 25 becomes

$$
\frac{\partial p}{\partial \hat{n}}=i \rho \omega \mathbf{u} .
$$

\subsection{Green's Function}

Once the boundary conditions are known, a Green's function that satisfies the inhomogeneous Helmholtz equation $\left(\nabla^{2}+k^{2}\right) G\left(\mathbf{r} \mid \mathbf{r}_{\mathbf{0}}\right)=\delta\left(\mathbf{r}-\mathbf{r}_{\mathbf{0}}\right)$ may be defined as

$$
G\left(\mathbf{r} \mid \mathbf{r}_{\mathbf{0}}\right)=\frac{1}{4 \pi} \frac{e^{j \mathbf{k} \cdot\left(\mathbf{r}-\mathbf{r}_{\mathbf{0}}\right)}}{\left|\mathbf{r}-\mathbf{r}_{\mathbf{0}}\right|}
$$

This is known as the free-space Green's function. Physically, it describes the field value at any point due to excitation by a single point source. For multiple or distributed sources, the principle of superposition applies and the resultant field may be found by summation or integration.

\subsection{Scattering Theory}

\section{Kirchhoff-Helmholtz Integral Theorem}


An exceptionally powerful tool for solving scattering problems is the Kirchhoff-Helmholtz integral theorem. The full derivation is not presented at this time, instead the reader is referred to Blackstock [5, p. 473] or Pierce [4, p. 182]. In its most general form, the theorem states that the pressure $p$ at a field point $\mathbf{r}_{\mathbf{0}}$ is described by

$$
p\left(\mathbf{r}_{\mathbf{0}}\right)=\int_{S}\left[p\left(\mathbf{r}_{\mathbf{0} S}\right) \nabla G\left(\mathbf{r}_{0} \mid \mathbf{r}_{S}\right)-G\left(\mathbf{r}_{0} \mid \mathbf{r}_{\mathbf{S}}\right) \nabla p\left(\mathbf{r}_{S}\right)\right] \cdot \mathbf{n}_{S} d S
$$

where $\mathbf{n}_{S}$ is the surface normal of the scattering object and $\mathbf{r}_{S}$ corresponds to the surface position. As before, the equation is simplified in the time-harmonic case. Using Euler's equation to relate the gradient of pressure to the normal particle velocity and remembering that shear forces are negligible, equation 28 becomes

$$
p\left(\mathbf{r}_{\mathbf{0}}\right)=\int_{S}\left[p\left(\mathbf{r}_{S}\right) \frac{\partial}{\partial n_{S}} G\left(\mathbf{r}_{0} \mid \mathbf{r}_{S}\right)-i \omega \rho_{0} \mathbf{u}\left(\mathbf{r}_{S}\right) G\left(\mathbf{r}_{0} \mid \mathbf{r}_{S}\right)\right] d S .
$$

The Kirchoff-Helmholtz integral theorem uses the appropriate Green's function to relate the total field to the pressure and particle velocity on the surface of the scatterer.

\section{Series Solutions}

An alternative method of solving the problem is the use of series. This method may be more simple in certain coordinates, but is less general than the integral method. In this approach, the incident plane wave is expanded in a series of spherical Bessel functions.

$$
p_{i}=A \sum_{n=0}^{\infty} \sum_{m=-n}^{n} 4 \pi i^{n} \overline{Y_{n}^{m}\left(\phi_{k}, \theta_{k}\right)} j_{n}(k r) Y_{n}^{m}(\phi, \theta)
$$

where $\phi_{k}$ and $\theta_{k}$ indicate the direction of propagation of the incident plane wave and the overbar denotes the complex conjugate. It is also assumed that the scattered field $p_{s}$ and the internal field of the scatterer $p_{d}$ can be expressed in an identical manner,

$$
p_{s}=\sum_{n=0}^{\infty} \sum_{m=-n}^{n} a_{m, n} h_{n}^{(1)}(k r) Y_{n}^{m}(\phi, \theta)
$$

and

$$
p_{d}=\sum_{n=0}^{\infty} \sum_{m=-n}^{n} b_{m, n} j_{n}(k r) Y_{n}^{m}(\phi, \theta) .
$$

Since the scattered field must satisfy the Sommerfeld radiation condition, the spherical Hankel function must be used to express the scattered field. In contrast, the interior field requires the use of the spherical Bessel to satisfy the boundedness condition. 
By using the boundary conditions established previously, it is possible to solve for the coefficients $a_{m, n}$ and $b_{m, n}$. Due to the orthogonality of the spherical harmonics, they may be equated term by term to find that

$$
\begin{gathered}
a_{0,0}=\frac{2 A \sqrt{\pi}\left[k_{d} \rho j_{0}^{\prime}\left(k_{d} a\right) j_{0}(k a)-k \rho_{d} j_{0}^{\prime}(k a) j_{0}\left(k_{d} a\right)\right]-k_{d} \rho j_{0}^{\prime}\left(k_{d} a\right) \Delta p}{k \rho_{d} h_{0}^{(1) \prime}(k a) j_{0}\left(k_{d} a\right)-k_{d} \rho h_{0}^{(1)}(k a) j_{0}^{\prime}\left(k_{d} a\right)}, \\
a_{m, n}=4 A \pi i^{n} \frac{Y_{n}^{m}\left(\phi_{k}, \theta_{k}\right)}{k_{d} \rho j_{n}^{\prime}\left(k_{d} a\right) j_{n}(k a)-k \rho_{d} j_{0}^{\prime}(k a) j_{0}\left(k_{d} a\right)} \frac{k_{d}^{(1) \prime}(k a) j_{n}\left(k_{d} a\right)-k_{d} \rho h_{n}^{(1)}(k a) j_{n}^{\prime}\left(k_{d} a\right)}{k \rho_{n}^{(1)}}
\end{gathered}
$$

for $m, n \neq 0$,

$$
b_{0,0}=\frac{2 \pi A j_{0}(k a)+a_{0,0} h^{(1) \prime}-\Delta p}{j_{0}\left(k_{d} a\right)}
$$

and

$$
b_{m, n}=\frac{\left.4 A \pi i^{n} j_{n}^{(} k a\right) \overline{Y_{n}^{m}\left(\phi_{k}, \theta_{k}\right)}+a_{m, n} h_{n}^{(1)}(k a)}{j_{n}^{\prime}\left(k_{d} a\right)} .
$$

for $m, n \neq 0$. These coefficients may be used to obtain plots of the total pressure field similar to those in Figures 5 and 6.

\section{Discussion and Conclusion}

The similarities between the results obtained from experimental, numerical, and analytical results are apparent. The numerical results agree with the experimental results only on a qualitative level due to the number of dimensions considered. Despite this limitation, the overall features of the field are strikingly similar in the number and location of the nodes. The analytical results agree very closely with the experiment, especially in the angular location of the nodes. This agreement suggests that the analytical model suggested is a valid representation of the phenomenon.

The balloon lens presents an intriguing demonstration for students of all levels. Although the experimental results may be surprising when compared to the prediction based on a ball lens or ray tracing, the main features of the field are similar enough that the lens description provides a valid first-order approximation. Indeed, the "focal" region is significant enough that an unaided ear can localize it. Thus for beginning physics students, it provides an excellent hands-on experiment and an opportunity to think laterally between the familiar optical domain and the acoustic domain. The same demonstration may provide undergraduate physics students with an opportunity to compare a numerical model to experimental observations. It also provides students learning about scattering with a simple, hands-on, macroscopic demonstration of scattering.

\section{References}

[1] Philip M. Morse and K. Uno Ingard. Theoretical Acoustics. Princeton University Press, Princeton, NJ, 1986. 
[2] Rainer Kress. Acoustic Scattering, Scattering and Inverse Scattering in Pure and Applied Science. Academic Press, London, 2001.

[3] Mary L. Boas. Mathematical Methods in the Physical Sciences. John Wiley and Sons, Inc., Hoboken, NJ, third edition, 2006.

[4] Allan D. Pierce. Acoustics: An Introduction to Its Physical Principles and Applications. Acoustical Society of America, Melville, NY, 1989.

[5] David T. Blackstock. Physical Acoustics. John Wiley and Sons, Inc., New York, NY, 2000. 


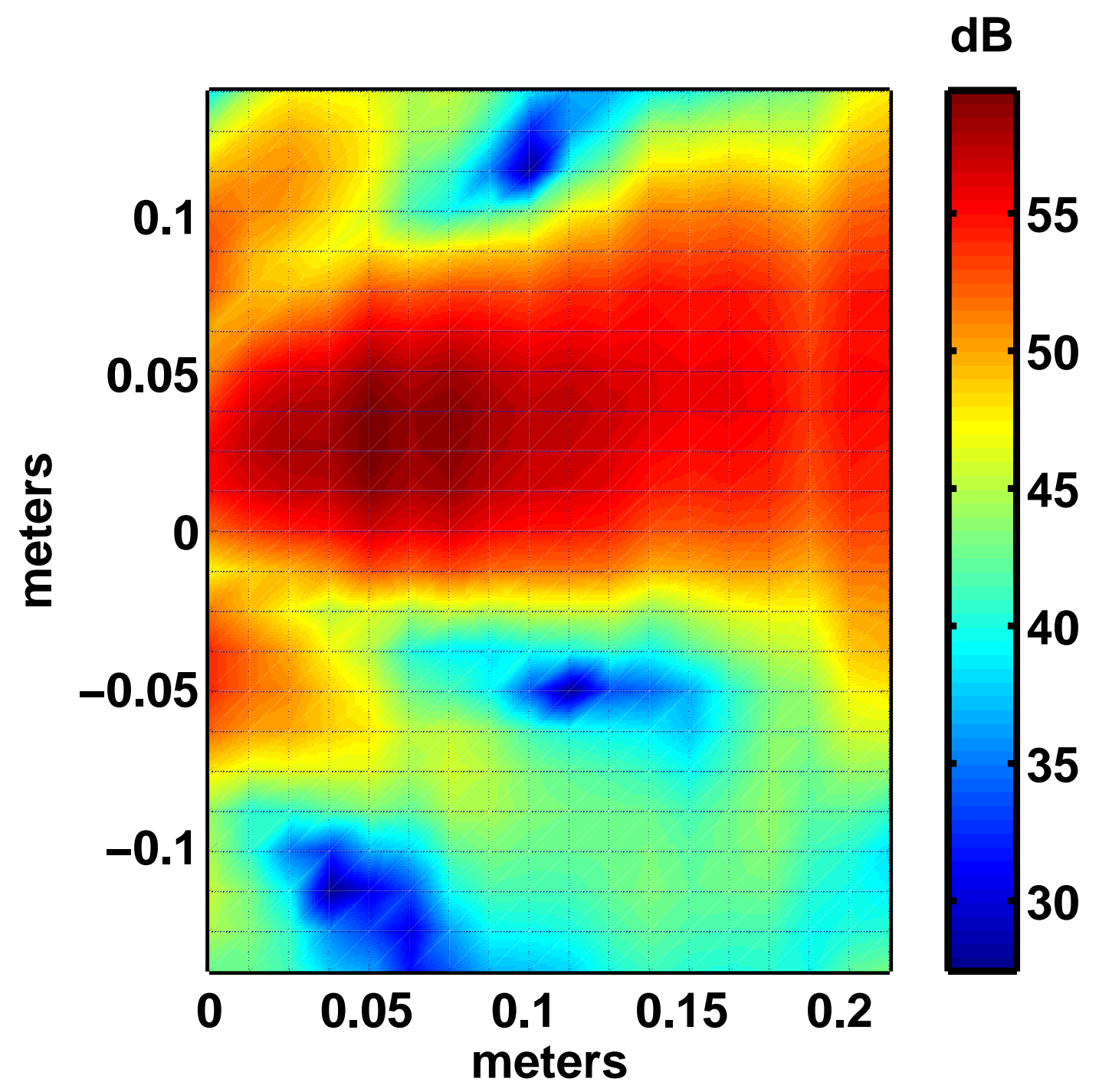

Fig. 1: Region $0.22 \mathrm{~m}$ by $0.3 \mathrm{~m}$ in front of balloon at approximately $3000 \mathrm{~Hz}$ as observed experimentally. 


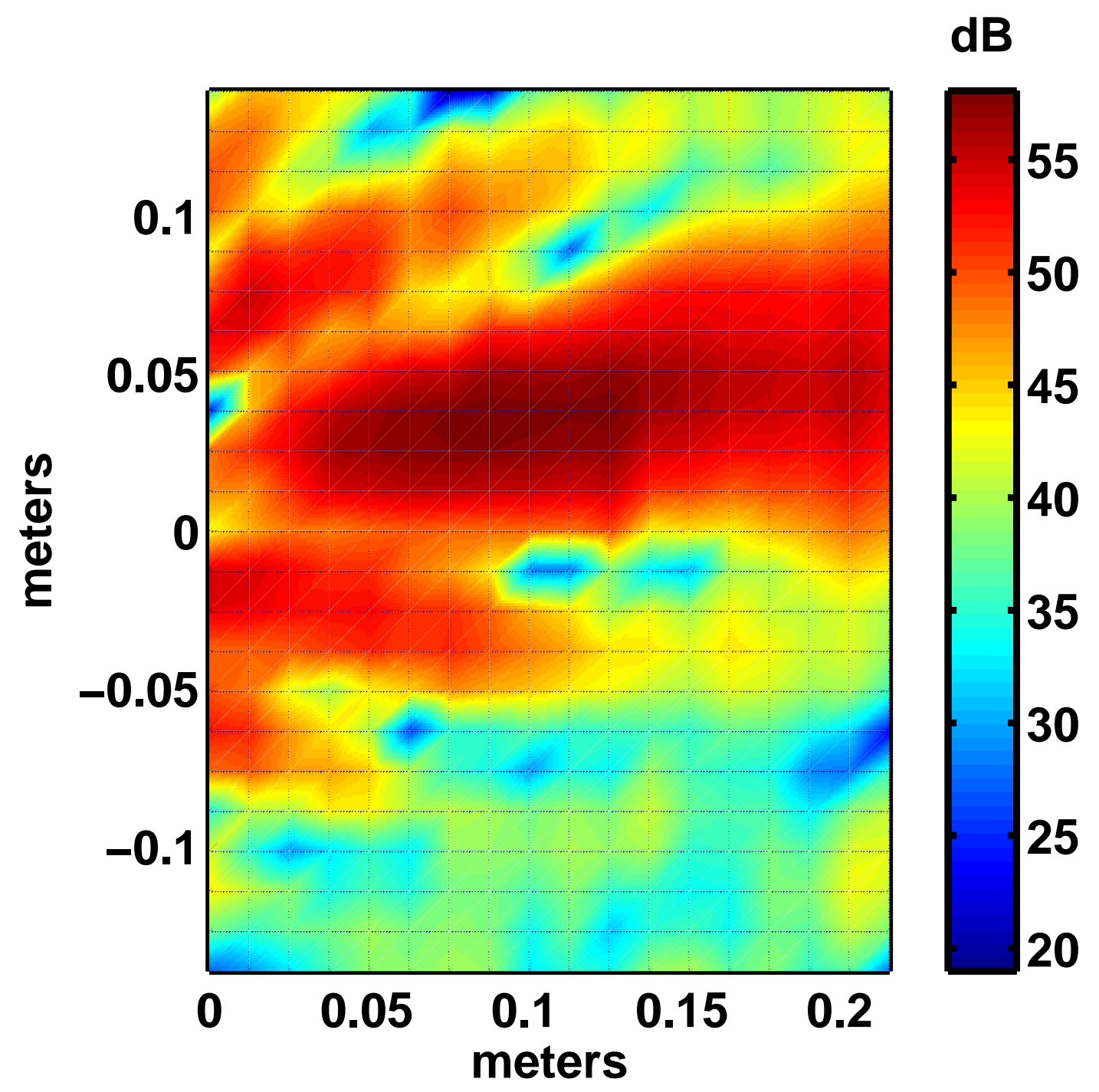

Fig. 2: Region $0.22 \mathrm{~m}$ by $0.3 \mathrm{~m}$ in front of balloon at approximately $5000 \mathrm{~Hz}$ as observed experimentally. 


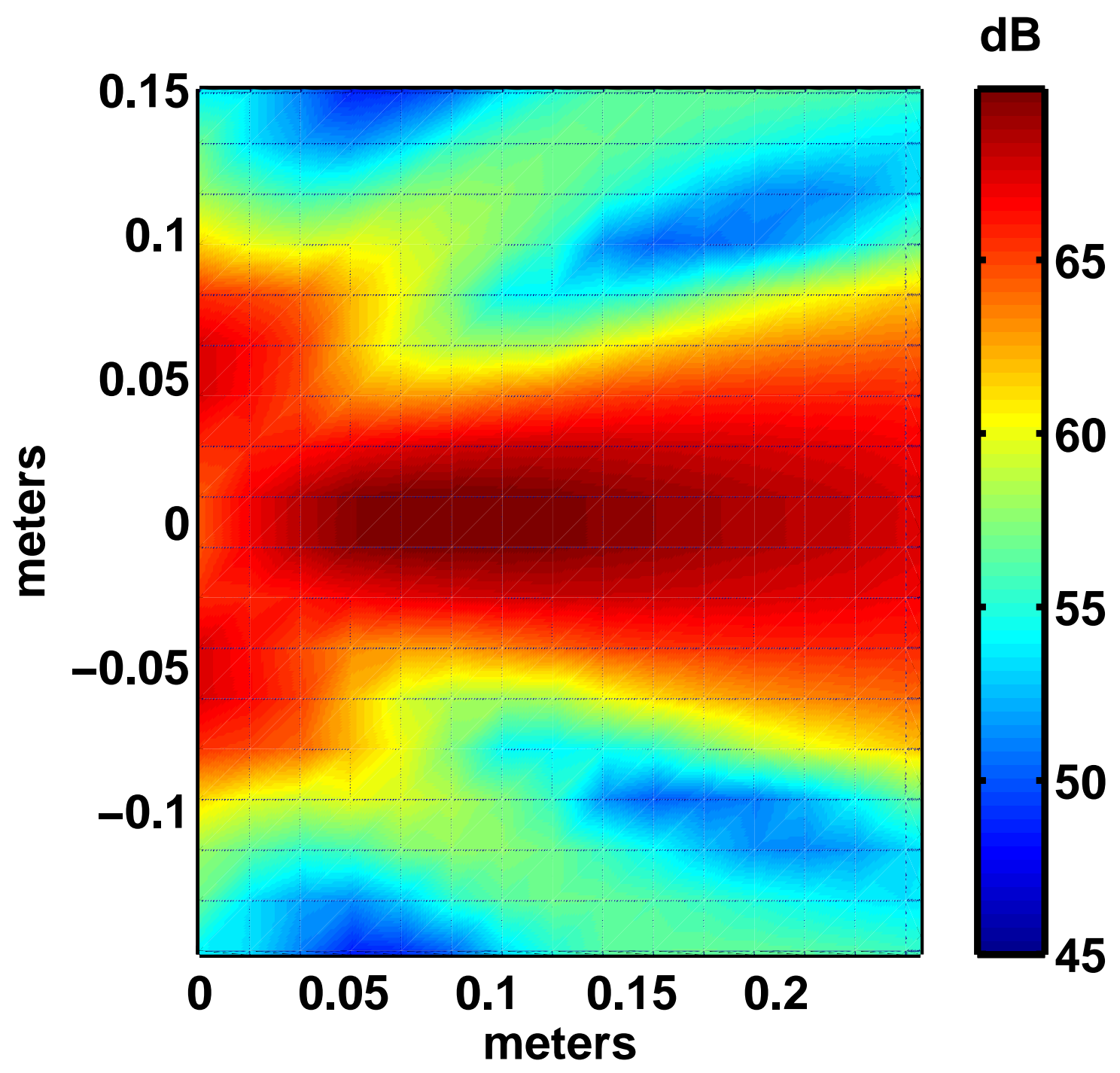

Fig. 3: Region $0.22 \mathrm{~m}$ by $0.3 \mathrm{~m}$ in front of balloon at approximately $3000 \mathrm{~Hz}$ as predicted by a staggered leapfrog method. 


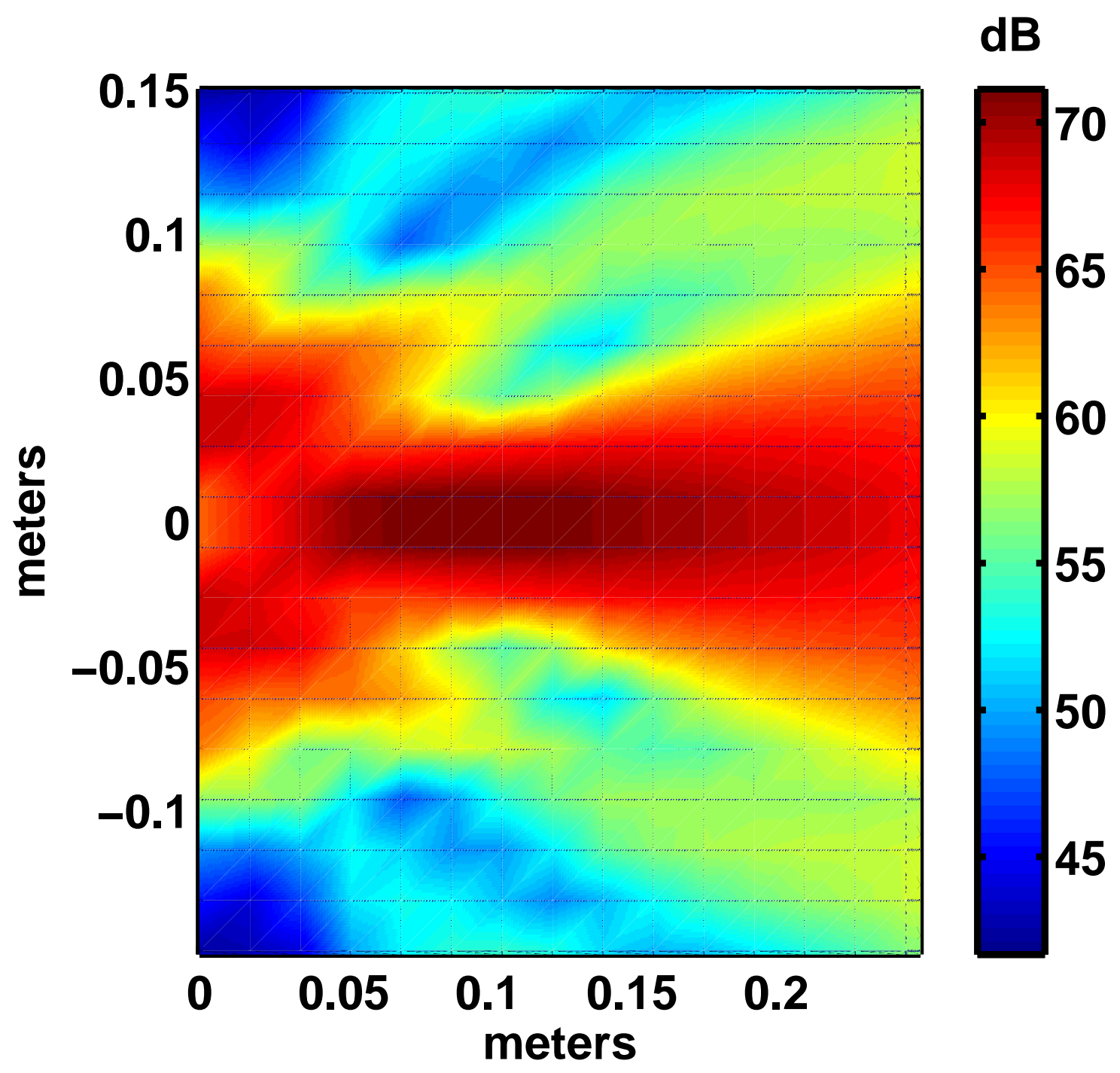

Fig. 4: Region $0.22 \mathrm{~m}$ by $0.3 \mathrm{~m}$ in front of balloon at approximately $5000 \mathrm{~Hz}$ as predicted by a staggered leapfrog method. 


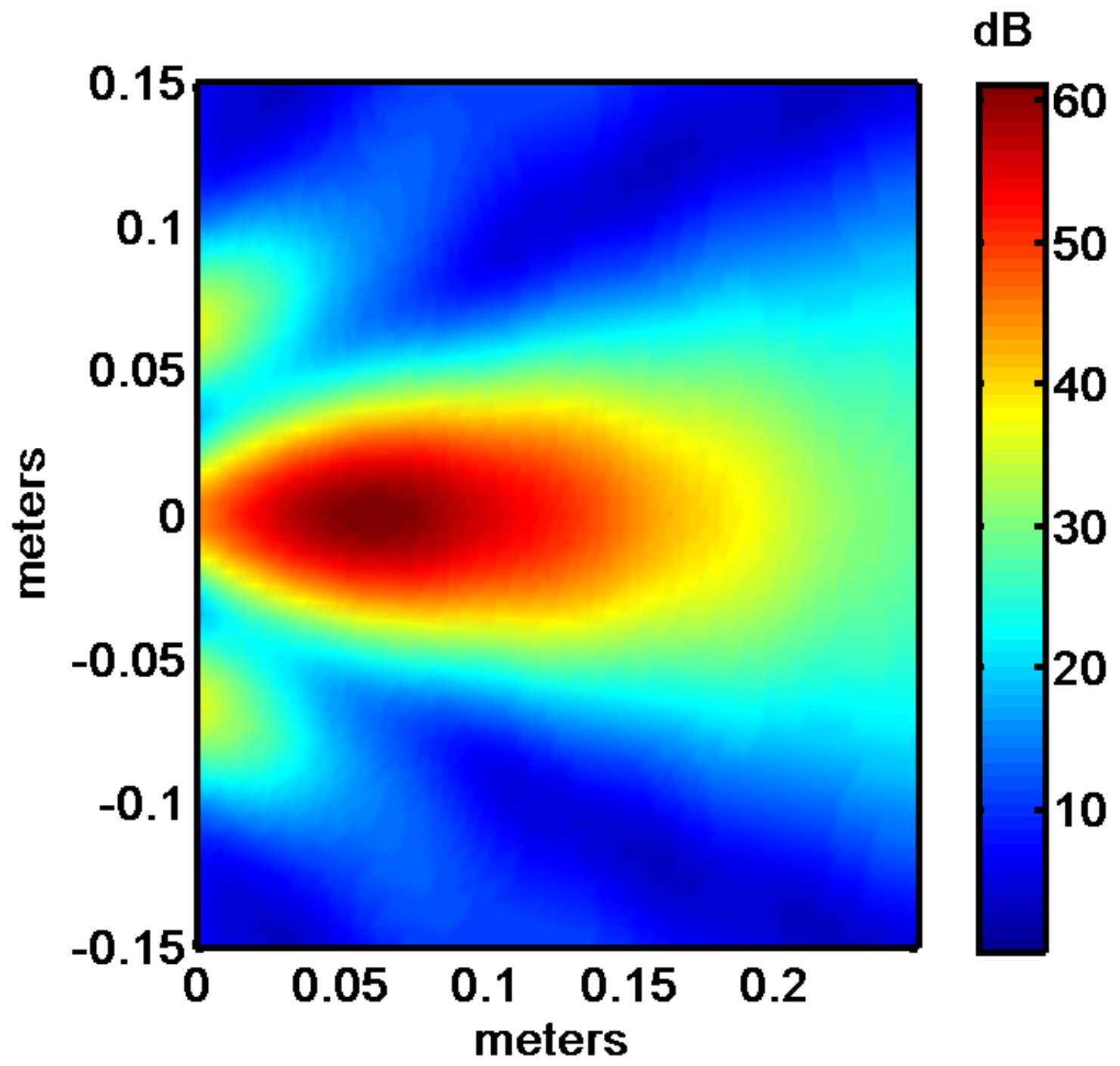

Fig. 5: Region $0.22 \mathrm{~m}$ by $0.3 \mathrm{~m}$ in front of balloon at $3000 \mathrm{~Hz}$ as predicted using an analytical series solution. 


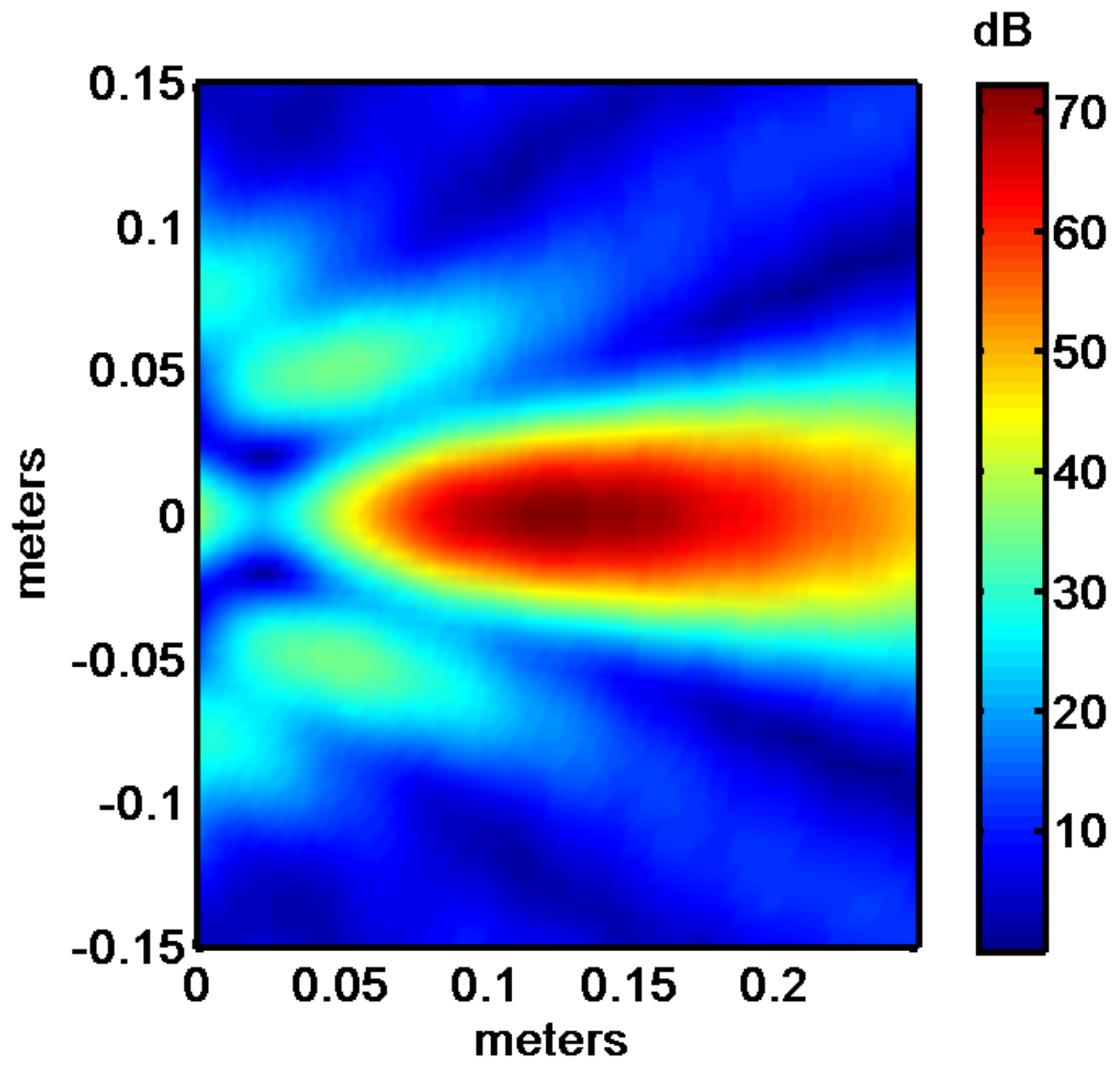

Fig. 6: Region $0.22 \mathrm{~m}$ by $0.3 \mathrm{~m}$ in front of balloon at $5000 \mathrm{~Hz}$ as predicted using an analytical series solution. 


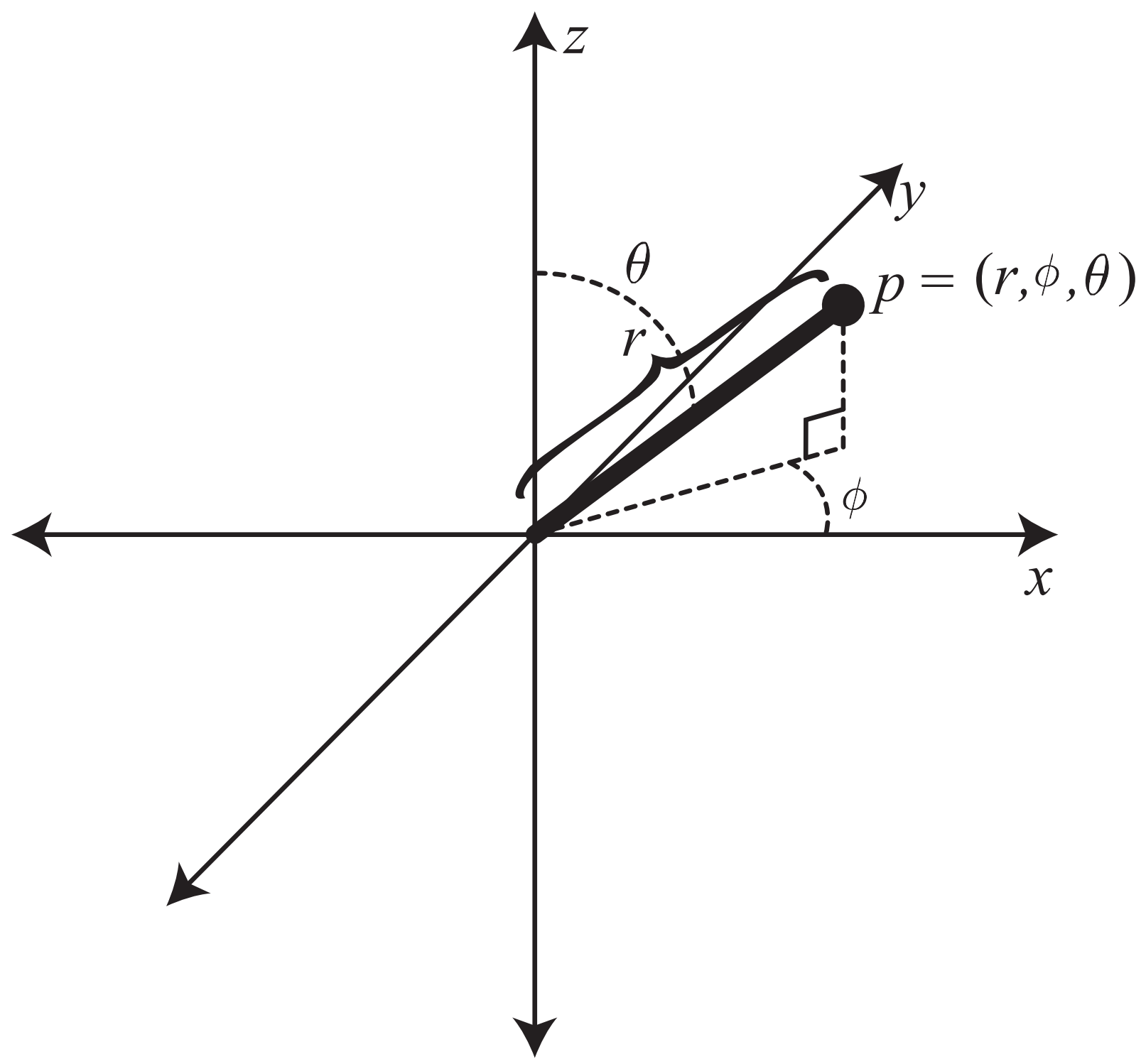

Fig. 7: Spherical Coordinate System 